\title{
Double-bound equivalent of the three-body Coulomb double-continuum wave function
}

\author{
L. U. Ancarani ${ }^{1}$ and G. Gasaneo ${ }^{2}$ \\ ${ }^{1}$ Laboratoire de Physique Moléculaire et des Collisions, Université Paul Verlaine-Metz, 57078 Metz, France \\ ${ }^{2}$ Departamento de Física, Universidad Nacional del Sur and Consejo Nacional de Investigaciones Científicas y Técnicas, \\ 8000 Bahía Blanca, Buenos Aires, Argentina
}

(Received 2 November 2006; revised manuscript received 21 December 2006; published 9 March 2007)

\begin{abstract}
In cross-section calculations of electron or photon double ionization processes of two-electron atoms, it is desirable to have a symmetric description of initial and final states. In this contribution, we search the doubly bound analog, for $S$ states, of the well-known and widely used three-body Coulomb (C3) double-continuum wave function. This is performed with two alternative approaches: through an analytic continuation of the C3 continuum and through a "C3 approach" of the Hylleraas equation for $S$ bound states. The double-bound analog consists of the product of two Coulomb bound states (one for each electron-nucleus interaction) multiplied by a Coulomb distortion factor which describes the electron-electron correlation. Our result differs from Pluvinage's wave function which is commonly misbelieved to be the bound counterpart of the C3 double continuum. With a rigorous treatment of the analytic continuation of the distortion factor, the double-bound equivalent of the $\mathrm{C} 3$ model is also found. Though the purpose is not to obtain good two-electron bound states, the found bound wave functions are tested, in the case of helium, through various local and mean quantities which probe different regions of the configuration space, in particular those close to the two-particle coalescence points.
\end{abstract}

DOI: 10.1103/PhysRevA.75.032706

PACS number(s): 34.80.Dp, 32.80.Fb, 31.25.-v

\section{INTRODUCTION}

In this contribution we are interested in the inter-relation between the description of the double-continuum final state and of the doubly bound initial state of two-electron atoms. This is particularly important in the theoretical study of the double ionization of helium, whether by electron $[(e, 3 e)$ experiments] [1] or photon $[(\gamma, 2 e)$ experiments] [2] impact. The full three-body Coulomb problem has been extensively studied, but no exact wave function is known for either the scattering or the bound states. Hence approximate wave functions are generally used when calculating double ionization cross sections. The choice of both the initial and final wave functions leads to different results [1,2] (see also recent discussions in [3-5]), so that it is important to try to understand the origin of these differences. Moreover, it is well known that having a symmetric description of the initial and final states is desirable since it avoids spurious contributions in the calculated cross sections. In this way, even relatively simple-but correlated-initial- and final-state wave functions can yield calculated cross sections in agreement with experimental data (see, e.g., the recent study of $(e, 3 e)$ processes $[4,6])$. This, however, is true as long as the wave functions include correlation in a "similar" way (we shall return to this point below).

In this report, we investigate the issue of constructing symmetrically double-continuum and double-bound approximate solutions for two-electron atoms. Generally speaking, this is not an easy task. Suppose, for example, that-in order to improve the description of a two-electron state-one introduces static (dynamic) screening effects in a bound (continuum) trial wave function: how can one modify the continuum (bound) wave function to include these effects to the same extent (or in a similar way)? Since there is no straight answer to this question, we have considered the "relatively simple" case of the well-known and widely used doublecontinuum three-body Coulomb (C3) wave function [7] (also called $3 \mathrm{C}$ or BBK model). The main aim is to find its doubly bound equivalent (for $S$ states) in order to have, for the first time, a symmetric description of correlated bound and continuum wave functions. This, curiously, has not been presented in the literature. The reason is possibly found in the common misbelief that the helium Pluvinage wave function $[8]$ is the answer (see, e.g., $[5,6,9]$ ). We shall show here that, while the mathematical approaches in both the continuum and bound cases present some similarities, this is not the case.

Though it has known shortcomings and kinematical limitations in applicability, the $\mathrm{C} 3$ wave function has been, and is currently, successfully used for calculating cross sections for single and double ionization, amongst other processes $[1,2,10]$. The C3 wave function is an approximate model describing the dynamics of three charged particles. In the case of two electrons in the field of a nucleus, it consists of the product of two Coulomb wave functions (one for each electron-nucleus interaction) multiplied by a distortion factor which describes the electron-electron correlation. The strength of the $\mathrm{C} 3$ continuum lies in the fact that it contains a large part of the multiple-scattering terms in the three twobody Coulomb potentials. Moreover, by construction, the $\mathrm{C} 3$ wave function (i) satisfies Kato's cusp conditions at all twobody coalescence points [11] (it fails, however, to satisfy the triple coalescence condition; see, e.g., Ref. [12]); (ii) it has the correct asymptotic behavior when all interparticle distances are large (so-called region $\Omega_{0}$ ). The main shortcomings, on the other hand, are the failure in describing the proper behavior: (i) at intermediate interparticle distances; (ii) when one particle is far away from the other two. Moreover, because of its asymptotic nature, its range of applicability is restricted to sufficiently large momenta. In order to correct some of these deficiencies, several improvements of 
the original C3 double-continuum wave function can be found in the literature. Most of them, built preserving its simple mathematical form, introduce coordinate -or velocitydependent effective charges (see, e.g., [1,9,13-19]), thus including dynamical screening. However, the choice of effective charges - though submitted to physical constraints-is arbitrary. According to the kinematical conditions of a double ionization process, the resulting cross sections can be either better or worse than those obtained with the original C3 wave function (see, e.g., Refs. [9,20-22]). Moreover, it is difficult to justify a different choice of effective charges for varying kinematical experimental conditions.

The original C3 wave function has been used in combination with several trial ground-state wave functions, for differential cross-section calculations of double ionization processes, in particular for helium. Recently, curious results have been observed for high-energy $(e, 3 e)$ processes: the use of sophisticated bound wave functions yield an agreement with experimental data which is worse than when relatively simple helium wave functions are used [3-5,23]. In particular, the rather simple Pluvinage wave function [8] gives good agreement [6] with the absolute experimental data of [21]; this was partly attributed to the fact that "the Pluvinage wave function is the doubly bound analog of the C3 wave function" [6] (and so the initial state is treated in equal manner to the final state). Practically the same level of agreement was subsequently found also with other simple bound wave functions [4,24]. In constructing Pluvinage-type wave functions with a superposition of configurations, it was observed that the agreement is lost $[5,25]$. The explanation of these results is found in the way the correlation is introduced in both initial and final states. Only when radial and angular (by angular we mean interparticle dependent only) correlations are not mixed in the initial state-similarly to the C3 continuum-agreement with high-energy $(e, 3 e)$ differential cross sections is found.

As the initial state is concerned there exist in the literature many trial wave functions (in the case of helium, some reaching extremely good energy levels; see, e.g., [26], and references therein). According to the variational principle, by adding more terms (and hence more variational parameters) it is possible to improve the ground-state energy. Trial wave functions can be constructed either with terms of a chosen mathematical-and presumably good-form or using the principle of superposition of configuration with a suitable basis set (in the latter case, one may systematically increase the number of terms). Screening effects can be included either directly (by using effective charges) or indirectly (for example, through a superposition of configurations). Contrary to the bound state, the construction of a mathematical final state is much more delicate. Only few closed forms for the correlated double continuum are known, and the systematic improvement by addition of more terms is not at all obvious, and quickly becomes cumbersome. One of the most used final states is the C3 wave function. Besides the improvement through the introduction of effective charges, some attempts to go beyond the C3 model, including higher orders of Coulomb-like wave functions, have also been reported (see, e.g., [27-30], and references therein).

Let us now return to the issue of having a symmetrical description of initial and final states. While it is relatively easy to improve the initial double bound state, one should improve the double-continuum state in an equal manner, i.e., with a similar degree of correlation: this is generally not feasible. As mentioned above, in this contribution, we consider the C3 wave function and search its bound equivalent for $S$ states. The result can then be used as a starting point from which to go further. For example, with a superposition of configuration of these equivalent bound $S$ states, one can envisage to construct - in a systematic way-better bound states which are still of the same family (work in preparation). This is exactly the approach considered recently with Pluvinage-type wave functions [5,25]: however, as mentioned above, Pluvinage's wave function is not the bound analog of the $\mathrm{C} 3$ continuum, and hence these studies were based on a starting point which is wrong if one aims at a symmetric description.

Since the C3 model was first introduced in the literature, its different versions have been widely used as final-state wave function to calculate cross sections for single and double ionization processes whether by electron, proton, or photon impact. We have already mentioned that, in collision calculations with approximate solutions, it is desirable to have a symmetric description of the initial and final states. The answer in the case of C3-like wave functions and its application, for example, to $(e, 3 e)$ processes, could help to elucidate the discrepancies observed on the calculated cross sections [4-6,9,20,21]. While the double-bound counterpart of the continuum $\mathrm{C} 3$ wave function is absent from the literature, we should mention-for completeness-that, in the case where two particles of a three-particle system form a bound compound, C3-like wave functions have been reported. Dewangan [31] developed an approach adequate for the process of electron-impact excitation of hydrogen: one of the electron-nucleus Coulomb wave function is replaced by a bound atomic eigenfunction, and the momentum corresponding to the electron-nucleus motion is set to zero. A much more mathematically careful study of this situation has been presented by Berakdar [32] and later on by Garibotti et al. [33]. In Berakdar's work, the author has given an asymptotically correct solution for the case where two of the particles are bound in any arbitrary state while the third has an arbitrary momentum. On the other hand, Garibotti et al. have derived the $\mathrm{C} 3$ analog corresponding to this situation by performing, on the double-continuum $\mathrm{C} 3$, the analytic continuation to the complex plane of one of the particles' relative momentum.

We would like to point out that there is no intention here to obtain good two-electron wave functions for $S$ states. The aim is to find the double-bound analog of the C3 wave function, in order to have a truly symmetric description of the double bound and double continuum. The result can be considered as a starting point for further improvements.

To achieve our aim, we organize the paper as follows. We need first to briefly describe how the C3 wave function corresponding to positive energy is constructed, and indicate which approximate Hamiltonian it effectively satisfies and what terms are neglected from the three-body Hamiltonian (Sec. II). We then search the doubly bound analog (for $S$ states) of the C3 double-continuum wave function in Sec. III. Two different approaches are used: through an analytic con- 
tinuation (Sec. III A) and through a "C3 approach" but for the bound $S$ states Schrödinger equation (Sec. III B). The second derivation allows us to show which terms of the Hylleraas Hamiltonian (see, e.g., [4]) are neglected to obtain the doubly bound analog of the $\mathrm{C} 3$ double continuum. The bound function obtained from either of the two procedures corresponds to the product of two Coulomb bound states multiplied by a Coulomb distortion factor; thus the bound C3 satisfies too all the cusp conditions at the two-body coalescence points. In Sec. IV, the bound electron-electron distortion factor is discussed in more detail: a simple approach (Sec. IV A) yields a bound analog, while a rigorous approach (Sec. IV B) gives the doubly bound equivalent sought after. For illustration, the mean and local energies for the found wave functions are compared in the case of the ground state of helium. The behavior of the bound states near the twoparticle coalescence points is also studied (Sec. V) through dynamical quantities (associated to photoabsorption and Compton scattering) which probe various parts of the configuration space. A summary of our findings, and perspectives for future work are presented in Sec. VI. In what follows, atomic units are used throughout.

\section{C3 DOUBLE-CONTINUUM WAVE FUNCTION}

Let us start from the nonrelativistic Schrödinger equation

$$
H \Psi=E \Psi,
$$

corresponding to two electrons in the presence of a nucleus of charge $Z$. For notation simplicity, we consider the nucleus to be infinitely heavy and fixed at the origin of coordinates. Let $\mathbf{r}_{1}$ and $\mathbf{r}_{2}$ represent the two electrons positions and $\mathbf{r}_{12}$ $=\mathbf{r}_{1}-\mathbf{r}_{2}$ the electron-electron relative vector. In terms of the interparticles coordinates $\mathbf{r}_{1}, \mathbf{r}_{2}, \mathbf{r}_{12}$ (labeled 1, 2, and 12, respectively), the Hamiltonian reads

$$
\begin{aligned}
H= & \left(-\frac{1}{2} \nabla_{1}^{2}+V_{1}\right)+\left(-\frac{1}{2} \nabla_{2}^{2}+V_{2}\right) \\
& +\left(-\nabla_{12}^{2}+V_{12}\right)+\left(\nabla_{2} \nabla_{12}-\nabla_{1} \nabla_{12}\right),
\end{aligned}
$$

where

$$
\begin{gathered}
V_{j}=\frac{Z z_{j}}{r_{j}}(j=1,2), \\
V_{12}=\frac{z_{1} z_{2}}{r_{12}}
\end{gathered}
$$

are the Coulomb potentials $\left(z_{1}=z_{2}=-1\right)$. It is worth reminding that $\mathbf{r}_{12}$ does depend on the vectors $\mathbf{r}_{1}$ and $\mathbf{r}_{2}$, even though in the Hamiltonian the three variables seem to be independent. The last bracket in the definition of the Hamiltonian $H$ corresponds to the kinetic operator, named crossed term hereafter, which mixes the coordinates and gives the nonseparable character to the three-body system. Whether the energy $E$ of the system is positive or negative, no closedform solution for $\Psi$ is known up to now.

Consider the case of the double continuum, where the two electrons have momenta $\mathbf{k}_{1}$ and $\mathbf{k}_{2}$, respectively. Without loss of generality, let us start by writing

$$
\Psi=e^{i \mathbf{k}_{1} \cdot \mathbf{r}_{1}} e^{i \mathbf{k}_{2} \cdot \mathbf{r}_{2} \Phi} .
$$

Upon replacement into Schrödinger equation (1), one easily obtains the equation for $\Phi$

$$
\begin{aligned}
& {\left[\left(-\frac{1}{2} \nabla_{1}^{2}-i \mathbf{k}_{1} \cdot \nabla_{1}+V_{1}\right)+\left(-\frac{1}{2} \nabla_{2}^{2}-i \mathbf{k}_{2} \cdot \nabla_{2}+V_{2}\right)\right.} \\
& \quad+\left[-\nabla_{12}^{2}-i\left(\mathbf{k}_{1}-\mathbf{k}_{2}\right) \cdot \nabla_{12}+V_{12}\right]+\left(\frac{k_{1}^{2}+k_{2}^{2}}{2}-E\right) \\
& \left.\quad+\left(\nabla_{2} \cdot \nabla_{12}-\nabla_{1} \cdot \nabla_{12}\right)\right] \Phi=0 .
\end{aligned}
$$

Consider the energy of the system to be $E=\left(k_{1}^{2}+k_{2}^{2}\right) / 2$. If we neglect the crossed term, Eq. (5) becomes a separable differential equation. The three parts of the Hamiltonian are solved, respectively, by a Kummer confluent hypergeometric function [34] ${ }_{1} F_{1}(i \alpha, 1,-i(k r+\mathbf{k} \cdot \mathbf{r}))$, with the appropriate $k$ and $\alpha$. Collecting the results, we get the C3 doublecontinuum wave function

$$
\begin{aligned}
\Psi_{C 3}= & N_{C 3} e^{i \mathbf{k}_{1} \cdot \mathbf{r}_{1}} F_{1}\left(i \alpha_{1}, 1,-i\left(k_{1} r_{1}+\mathbf{k}_{1} \cdot \mathbf{r}_{1}\right)\right) \\
& \times e^{i \mathbf{k}_{2} \cdot \mathbf{r}_{2}}{ }_{1} F_{1}\left(i \alpha_{2}, 1,-i\left(k_{2} r_{2}+\mathbf{k}_{2} \cdot \mathbf{r}_{2}\right)\right) \\
& \times{ }_{1} F_{1}\left(i \alpha_{12}, 1,-i\left(k_{12} r_{12}+\mathbf{k}_{12} \cdot \mathbf{r}_{12}\right)\right)
\end{aligned}
$$

where

$$
\begin{gathered}
\mathbf{k}_{12}=\left(\mathbf{k}_{1}-\mathbf{k}_{2}\right) / 2, \\
\alpha_{j}=\frac{Z z_{j}}{k_{j}} \quad(j=1,2), \\
\alpha_{12}=\frac{z_{1} z_{2}}{2 k_{12}} .
\end{gathered}
$$

The overall multiplying factor defined in [7] is

$$
\begin{aligned}
N_{C 3}= & e^{-(\pi / 2) \alpha_{1}} \Gamma\left(1-i \alpha_{1}\right) e^{-(\pi / 2) \alpha_{2}} \Gamma\left(1-i \alpha_{2}\right) e^{-(\pi / 2) \alpha_{12}} \\
& \times \Gamma\left(1-i \alpha_{12}\right)
\end{aligned}
$$

where $\Gamma(z)$ represents the Gamma function [34]. The $\Psi_{C 3}$ is the product of two plane waves (one for each electron) multiplied by three distortion factors $D(\alpha, \mathbf{k}, \mathbf{r})$ (the confluent hypergeometric function) corresponding to the three Coulomb interactions. Alternatively, the $\Psi_{C 3}$ can be expressed in terms of the product of two continuum Coulomb wave functions $C(\alpha, \mathbf{k}, \mathbf{r})$ (for electrons 1 and 2 ) by a distortion factor (for the $\mathbf{r}_{12}$ part)

$$
\Psi_{C 3}=N_{C 3} C\left(\alpha_{1}, \mathbf{k}_{1}, \mathbf{r}_{1}\right) C\left(\alpha_{2}, \mathbf{k}_{2}, \mathbf{r}_{2}\right) D\left(\alpha_{12}, \mathbf{k}_{12}, \mathbf{r}_{12}\right) .
$$

If we define the approximate Hamiltonian $H_{C 3}$ by

$$
\begin{aligned}
H_{C 3}= & \left(-\frac{1}{2} \nabla_{1}^{2}+V_{1}\right)+\left(-\frac{1}{2} \nabla_{2}^{2}+V_{2}\right) \\
& +\left[-\nabla_{12}^{2}-i\left(\mathbf{k}_{1}-\mathbf{k}_{2}\right) \cdot \nabla_{12}+V_{12}\right],
\end{aligned}
$$

$\Psi_{C 3}$ satisfies exactly the following equation: 


$$
H_{C 3} \Psi_{C 3}=E \Psi_{C 3} .
$$

The eigenvalue $E=\left(k_{1}^{2}+k_{2}^{2}\right) / 2$ and the wave function $\Psi_{C 3}$ are fully defined only through the momenta $\mathbf{k}_{1}$ and $\mathbf{k}_{2}$. Equation (9) is mathematically separable, and the solutions for the $\mathbf{r}_{1}$ and $\mathbf{r}_{2}$ parts are, in principle, unrelated to the $\mathbf{r}_{12}$ part. However, the solution of the $\mathbf{r}_{12}$ part is fixed by the momentum conservation $\left(\mathbf{k}_{1}-\mathbf{k}_{2}\right)$ and the external constraint given by Eqs. (7a) and (7c); in this sense therefore it is not fully independent of the $\mathbf{r}_{1}$ and $\mathbf{r}_{2}$ parts. Note also that the factor $D\left(\alpha_{12}, \mathbf{k}_{12}, \mathbf{r}_{12}\right)$ does not contribute to the energy, in agreement with the concept of distortion factor.

Explicitly, the term neglected in the full Schrödinger equation (1) reads

$$
\begin{aligned}
& \left(H-H_{C 3}\right) \Psi_{C 3}=\left[\left(\nabla_{2} \cdot \nabla_{12}-\nabla_{1} \cdot \nabla_{12}\right)+i\left(\mathbf{k}_{1}-\mathbf{k}_{2}\right) \cdot \nabla_{12}\right] \Psi_{C 3} \\
& =e^{i \mathbf{k}_{1} \cdot \mathbf{r}_{1}} e^{i \mathbf{k}_{2} \cdot \mathbf{r}_{2}}{ }_{1} F_{1}\left(i \alpha_{1}, 1,-i\left(k_{1} r_{1}+\mathbf{k}_{1} \cdot \mathbf{r}_{1}\right)\right) \\
& \times \nabla_{2{ }_{1}} F_{1}\left(i \alpha_{2}, 1,-i\left(k_{2} r_{2}+\mathbf{k}_{2} \cdot \mathbf{r}_{2}\right)\right) \\
& -{ }_{1} F_{1}\left(i \alpha_{2}, 1,-i\left(k_{2} r_{2}+\mathbf{k}_{2} \cdot \mathbf{r}_{2}\right)\right) \\
& \left.\times \nabla_{11} F_{1}\left(i \alpha_{1}, 1,-i\left(k_{1} r_{1}+\mathbf{k}_{1} \cdot \mathbf{r}_{1}\right)\right)\right] \\
& \times \nabla_{12}{ }_{1} F_{1}\left(i \alpha_{12}, 1,-i\left(k_{12} r_{12}+\mathbf{k}_{12} \cdot \mathbf{r}_{12}\right)\right) \text {. }
\end{aligned}
$$

This term depends on the $\Psi_{C 3}$ itself, and hence on the energy E.

The C3 function has been widely used in collision physics to describe the double continuum: it treats all Coulomb interactions on an equal footing and presents the correct asymptotic behavior when all interparticle distances are large (with the proper logarithmic phases which are the signature of Coulomb potentials) [7]. Moreover, by construction, the C3 function has also the correct behavior at the two-body coalescence points, and hence satisfies Kato's cusp conditions [11]. At the three-body coalescence it fails because it does not includes the appropriate logarithmic terms as can be seen from its power-series expansion [12].

In Sec. III B, we shall search for the doubly bound equivalent of $\Psi_{C 3}$, but starting from the bound $S$-state Hamiltonian. To do so, we shall use an alternative writing of $\Psi_{C 3}$. It is obtained by application of Kummer relation [see Eq. (13.1.27) of [34]] to the first two hypergeometric functions,

$$
\begin{aligned}
& { }_{1} F_{1}\left(i \alpha_{j}, 1,-i\left(k_{j} r_{j}+\mathbf{k}_{j} \cdot \mathbf{r}_{j}\right)\right) \\
& \quad=e^{-i\left(k_{j} r_{j}+\mathbf{k}_{j} \cdot \mathbf{r}_{j}\right)}{ }_{1} F_{1}\left(1-i \alpha_{j}, 1, i\left(k_{j} r_{j}+\mathbf{k}_{j} \cdot \mathbf{r}_{j}\right)\right) \quad(j=1,2),
\end{aligned}
$$

so that

$$
\begin{aligned}
\Psi_{C 3}= & N_{C 3} e^{-i k_{1} r_{1}} F_{1} F_{1}\left(1-i \alpha_{1}, 1, i\left(k_{1} r_{1}+\mathbf{k}_{1} \cdot \mathbf{r}_{1}\right)\right) \\
& \times e^{-i k_{2} r_{2}}{ }_{1} F_{1}\left(1-i \alpha_{2}, 1, i\left(k_{2} r_{2}+\mathbf{k}_{2} \cdot \mathbf{r}_{2}\right)\right) \\
& \times{ }_{1} F_{1}\left(i \alpha_{12}, 1,-i\left(k_{12} r_{12}+\mathbf{k}_{12} \cdot \mathbf{r}_{12}\right)\right) .
\end{aligned}
$$

Let us therefore consider again the Schrödinger equation (1) applied, this time, to

$$
\Psi=e^{-i k_{1} r_{1}} e^{-i k_{2} r_{2}} \varphi
$$

One gets

$$
\begin{aligned}
& {\left[\left(-\frac{1}{2} \nabla_{1}^{2}+i k_{1} \hat{\mathbf{r}}_{1} \cdot \nabla_{1}+i \frac{k_{1}}{r_{1}}+V_{1}\right)\right.} \\
& \quad+\left(-\frac{1}{2} \nabla_{2}^{2}+i k_{2} \hat{\mathbf{r}}_{2} \cdot \nabla_{2}+i \frac{k_{2}}{r_{2}}+V_{2}\right)+\left(-\nabla_{12}^{2}+V_{12}\right) \\
& \quad+\left(\frac{k_{1}^{2}+k_{2}^{2}}{2}-E\right)+\left(\nabla_{2} \cdot \nabla_{12}-\nabla_{1} \cdot \nabla_{12}\right) \\
& \left.+i\left(k_{1} \hat{\mathbf{r}}_{1}-k_{2} \hat{\mathbf{r}}_{2}\right) \cdot \nabla_{12}\right] \varphi=0,
\end{aligned}
$$

where the last term, named mixed term hereafter, also mixes the variables. One may recognize that the first two brackets are the differential equations for the functions ${ }_{1} F_{1}(1$ $\left.-i \alpha_{j}, 1, i\left(k_{j} r_{j}+\mathbf{k}_{j} \cdot \mathbf{r}_{j}\right)\right)(j=1,2)$. Hence we may take

$$
\begin{aligned}
\varphi= & { }_{1} F_{1}\left(1-i \alpha_{1}, 1, i\left(k_{1} r_{1}+\mathbf{k}_{1} \cdot \mathbf{r}_{1}\right)\right) \\
& \times{ }_{1} F_{1}\left(1-i \alpha_{2}, 1, i\left(k_{2} r_{2}+\mathbf{k}_{2} \cdot \mathbf{r}_{2}\right)\right) \chi,
\end{aligned}
$$

so that

$$
\Psi=C\left(\alpha_{1}, \mathbf{k}_{1}, \mathbf{r}_{1}\right) C\left(\alpha_{2}, \mathbf{k}_{2}, \mathbf{r}_{2}\right) \chi .
$$

Setting the energy $E=\left(k_{1}^{2}+k_{2}^{2}\right) / 2$, and assuming $\chi$ depends on the variable $\mathbf{r}_{12}$ only, Eq. (15) reduces to

$$
\begin{aligned}
& {\left[\left(-\nabla_{12}^{2}+V_{12}\right)+\left(\nabla_{2} \cdot \nabla_{12}-\nabla_{1} \cdot \nabla_{12}\right)+i\left(k_{1} \hat{\mathbf{r}}_{1}-k_{2} \hat{\mathbf{r}}_{2}\right) \cdot \nabla_{12}\right] \varphi} \\
& \quad=0
\end{aligned}
$$

(note that the operators apply to $\varphi$ and not to $\chi$ ). It does not appear from this equation that the remaining part $\chi$ of $\Psi$ should be the distortion factor $D\left(\alpha_{12}, \mathbf{k}_{12}, \mathbf{r}_{12}\right)$ like in Eq. (8). This is related to the fact that neglecting the crossed term at this stage is not the same as neglecting it when getting $\Psi_{C 3}$ from Eq. (5). This can be seen in the following way. Using Kummer relation (12) backwards, Eq. (18) becomes

$$
e^{-i k_{1} r_{1}} e^{-i k_{2} r_{2}}\left\{\frac{\varphi}{\chi}\left[-\nabla_{12}^{2}-i\left(\mathbf{k}_{1}-\mathbf{k}_{2}\right) \cdot \nabla_{12}+V_{12}\right]+Q\right\} \chi=0
$$

where the operator $Q$ reads

$$
\begin{aligned}
Q= & e^{i k_{1} r_{1}} e^{i \mathbf{k}_{1} \cdot \mathbf{r}_{1}} e^{i k_{2} r_{2}} e^{i \mathbf{k}_{2} \cdot \mathbf{r}_{2}}\left[{ }_{1} F_{1}\left(i \alpha_{1}, 1,-i\left(k_{1} r_{1}+\mathbf{k}_{1} \cdot \mathbf{r}_{1}\right)\right)\right. \\
& \times \nabla_{21} F_{1}\left(i \alpha_{2}, 1,-i\left(k_{2} r_{2}+\mathbf{k}_{2} \cdot \mathbf{r}_{2}\right)\right) \\
& -{ }_{1} F_{1}\left(i \alpha_{2}, 1,-i\left(k_{2} r_{2}+\mathbf{k}_{2} \cdot \mathbf{r}_{2}\right)\right) \\
& \left.\times \nabla_{11} F_{1}\left(i \alpha_{1}, 1,-i\left(k_{1} r_{1}+\mathbf{k}_{1} \cdot \mathbf{r}_{1}\right)\right)\right] \cdot \nabla_{12} \\
= & \left\{{ }_{1} F_{1}\left(1-i \alpha_{1}, 1, i\left(k_{1} r_{1}+\mathbf{k}_{1} \cdot \mathbf{r}_{1}\right)\right)\right. \\
& \times\left[\left(-i k_{2} \hat{\mathbf{r}}_{2}-i \mathbf{k}_{2}\right)+\nabla_{2}\right]_{1} F_{1}\left(1-i \alpha_{2}, 1, i\left(k_{2} r_{2}+\mathbf{k}_{2} \cdot \mathbf{r}_{2}\right)\right) \\
& -{ }_{1} F_{1}\left(1-i \alpha_{2}, 1, i\left(k_{2} r_{2}+\mathbf{k}_{2} \cdot \mathbf{r}_{2}\right)\right)\left[\left(-i k_{1} \hat{\mathbf{r}}_{1}-i \mathbf{k}_{1}\right)+\nabla_{1}\right] \\
& \left.\times{ }_{1} F_{1}\left(1-i \alpha_{1}, 1, i\left(k_{1} r_{1}+\mathbf{k}_{1} \cdot \mathbf{r}_{1}\right)\right)\right\} \cdot \nabla_{12} .
\end{aligned}
$$

[The second form of $Q$, Eq. (20b), is given here as it will be used in Sec. III B.] It is now clear that, if we neglect the 
action of operator $Q$ in Eq. (19) (i.e., exactly as we did when constructing $\Psi_{C 3}$ ) the remaining equation is solved by $\chi$ $=D\left(\alpha_{12}, \mathbf{k}_{12}, \mathbf{r}_{12}\right)$, and the $\mathrm{C} 3$ function is recovered [see Eq. (17)]. This point will be of relevance in Sec. III B.

Two more observations. If we neglect both the crossed and mixed operators in Eq. (18), we are left with the following differential equation for $\chi$ :

$$
\left(-\nabla_{12}^{2}+V_{12}\right) \chi=0 .
$$

The solution is a Coulomb function $C\left(1 /(2 k), \mathbf{k}, \mathbf{r}_{12}\right)$ with $k \rightarrow 0$ (arbitrary direction $\hat{\mathbf{k}}$ ), i.e., a Bessel function [see Eq. (13.3.1) of [34]]

$$
I_{0}\left(\sqrt{2\left(r_{12}+\hat{\mathbf{k}} \cdot \mathbf{r}_{12}\right)}\right) .
$$

The approximate solution for the double continuum is then

$$
\Psi=N\left(k_{12} \rightarrow 0\right) C\left(\alpha_{1}, \mathbf{k}_{1}, \mathbf{r}_{1}\right) C\left(\alpha_{2}, \mathbf{k}_{2}, \mathbf{r}_{2}\right) I_{0}\left(\sqrt{2\left(r_{12}+\hat{\mathbf{k}} \cdot \mathbf{r}_{12}\right)}\right) .
$$

It is interesting to note that the limit $k_{12} \rightarrow 0$ of the $\mathrm{C} 3$ function (6) gives the same answer.

If, on the other hand, we had ignored the electron-electron interaction from the outset, there would be no dependence on $\mathbf{r}_{12}$. The corresponding independent particles model for the continuum wave function is the product of the Coulomb wave functions [i.e., Eq. (17) where the correlation function $\chi=1]$ with the same energy $E=\left(k_{1}^{2}+k_{2}^{2}\right) / 2$.

\section{DOUBLY BOUND ANALOG OF THE C3 DOUBLE- CONTINUUM FUNCTION}

In this section we look for the doubly bound analog of the C3 double-continuum function. We shall proceed in two quite different ways which give the same answer. First we use the analytic continuation (Sec. III A) and then use a "C3 approach" for $S$ bound states (Sec. III B). To make the notation more explicit, in what follows the superscript "B" will be added whenever a bound state is involved. On the other hand, the subscript " $\mathrm{C} 3$ " will be maintained to remind that a quantity originates from the $\mathrm{C} 3$ continuum.

\section{A. Analytic continuation}

We can find the doubly analog of the $\mathrm{C} 3$ by performing its analytic continuation. We shall focus here on the $S$ bound states only. Let us first expand each term of the $\Psi_{C 3}$ wave function (8) in partial waves,

$$
\begin{aligned}
\Psi_{C 3}= & \left(\sum_{l_{1}} R_{l_{1}, k_{1}}\left(r_{1}\right) P_{l_{1}}\left(\cos \theta_{1}\right)\right)\left(\sum_{l_{2}} R_{l_{2}, k_{2}}\left(r_{2}\right) P_{l_{2}}\left(\cos \theta_{2}\right)\right) \\
& \times\left(\sum_{l} A_{l_{12}, k_{12}}\left(r_{12}\right) P_{l_{12}}\left(\cos \theta_{12}\right)\right),
\end{aligned}
$$

where $P_{l}(\cos \theta)$ are Legendre polynomials, $\theta_{1}$ (respectively $\theta_{2}, \theta_{12}$ ) is the angle between $\mathbf{k}_{1}$ and $\mathbf{r}_{1}$ (respectively $\mathbf{k}_{2}$ and $\mathbf{r}_{2}$, and $\mathbf{k}_{12}$ and $\left.\mathbf{r}_{12}\right) . R_{l_{j}, k_{j}}\left(r_{j}\right)$ are the $l_{j}$ Coulomb partial waves $(j=1,2)$,

$$
\begin{aligned}
& R_{l_{j}, k_{j}}\left(r_{j}\right)=e^{-(\pi / 2) \alpha_{j}}\left(2 l_{j}+1\right) \frac{\Gamma\left(l_{j}+1-i \alpha_{j}\right)}{\Gamma\left(2 l_{j}+2\right)}\left(2 i k_{j} r_{j}\right)_{j}^{l_{j}} e^{-i k_{j} r_{j}} \\
& \times_{1} F_{1}\left(l_{j}+1-i \alpha_{j}, 2 l_{j}+2,2 i k_{j} r_{j}\right),
\end{aligned}
$$

and the $A_{l_{12}, k_{12}}$ are given by

$$
\begin{aligned}
& A_{l_{12}, k_{12}}\left(r_{12}\right)=e^{-(\pi / 2) \alpha_{12} \Gamma\left(1-i \alpha_{12}\right)} \frac{\Gamma\left(l_{12}+i \alpha_{12}\right)}{\Gamma\left(i \alpha_{12}\right)\left(2 l_{12}-1\right) ! ! l_{12} !} \\
& \times\left(-i k_{12} r_{12}\right)^{l_{12}} \\
& \times_{1} F_{1}\left(l_{12}+i \alpha_{12}, 2 l_{12}+2,-2 i k_{12} r_{12}\right) \text {. }
\end{aligned}
$$

For $S$-states of the two-electron system, we retain only the $l=0$ contribution of each expansion, and find

$$
\begin{aligned}
\Psi_{C 3}^{(l=0)}= & R_{0, k_{1}}\left(r_{1}\right) R_{0, k_{2}}\left(r_{2}\right) A_{0, k_{12}}\left(r_{12}\right) \\
= & N_{C 3} e^{-i k_{1} r_{1}}{ }_{1} F_{1}\left(1-i \alpha_{1}, 2,2 i k_{1} r_{1}\right) e^{-i k_{2} r_{2}} \\
& \times_{1} F_{1}\left(1-i \alpha_{2}, 2,2 i k_{2} r_{2}\right)_{1} F_{1}\left(i \alpha_{12}, 2,-2 i k_{12} r_{12}\right) .
\end{aligned}
$$

We can now perform the analytic continuation by replacing $k_{j}$ by $i Z z_{j} / n_{j}$, so that $i \alpha_{j}=n_{j}$, where $n_{j}=1,2,3, \ldots(j$ $=1,2)$ [33]. The two continuum Coulomb partial waves in $r_{1}$ and $r_{2}$ get transformed into the product $\varphi_{n_{1}}\left(r_{1}\right) \varphi_{n_{2}}\left(r_{2}\right)$ of two radial hydrogenic bound functions

$$
\varphi_{n}(r)=e^{-Z r / n} F_{1}\left(1-n, 2, \frac{2 Z r}{n}\right),
$$

of principal quantum numbers $n_{1}$ and $n_{2}$ (here we have explicitly set $\left.z_{1}=z_{2}=-1\right)$. The energy $E$ of the system becomes $E^{B}=-\frac{Z^{2}}{2 n_{1}^{2}}-\frac{Z^{2}}{2 n_{2}^{2}}$. The corresponding Gamma factors $\Gamma\left(1-n_{j}\right)$, which are closely related to the Jost function, present a pole for any value of $n_{j}$. As in the case of the two-body Coulomb problem (see Ref. [33]), these poles (zeros of the $S$ matrix) remove the exponentially divergent part of the continuum wave function leading thus to the bound states $\varphi_{n}(r)$.

The issue remains of how to perform the analytic continuation of the third confluent hypergeometric function in Eq. (27). Indeed, the modulus

$$
k_{12}=\frac{1}{2}\left(k_{1}^{2}+k_{2}^{2}-2 \mathbf{k}_{1} \cdot \mathbf{k}_{2}\right)^{1 / 2}
$$

depends on $k_{1}, k_{2}$ and the angle between $\mathbf{k}_{1}$ and $\mathbf{k}_{2}$, and varies from $\left|k_{1}-k_{2}\right| / 2$ to $\left(k_{1}+k_{2}\right) / 2$. The following question arises: what should replace $k_{12}$ in Eq. (27)? We should remember that a well defined bound state must be independent of any quantization direction or spacial orientation of the reference system. The angular dependence in Eq. (28) needs a particular treatment which shall be given in Sec. IV B. For the moment, let us ignore the mathematical link between $k_{12}$ and $k_{1}, k_{2}$. Since $k_{1}$ and $k_{2}$ become imaginary by analytic continuation it is reasonable to expect the modulus $k_{12}$ to become imaginary as well. We can thus replace $k_{12}$ by a single value $i \kappa$. Based on this assumption, we can write down what can be considered as the doubly bound analog of the continuum $\mathrm{C} 3$ function, 


$$
\begin{aligned}
\Psi_{C 3}^{B}\left(n_{1}, n_{2}, \kappa\right)= & N_{C 3}^{B}\left(n_{1}, n_{2}, \kappa\right) \varphi_{n_{1}}\left(r_{1}\right) \varphi_{n_{2}}\left(r_{2}\right) \\
& \times{ }_{1} F_{1}\left(\frac{1}{2 \kappa}, 2,2 \kappa r_{12}\right)
\end{aligned}
$$

where $N_{C 3}^{B}\left[n_{1}, n_{2}, \kappa\right]$ is the normalization factor of the $S$ bound state. Independently of the choice of $\kappa$ (which will be discussed below, Sec. IV), the bound function (29) satisfies all two-body Kato cusp conditions.

\section{B. C3 approach for two-electron $S$ bound states}

We shall now adopt an approach similar to that used to find the C3, but for the $S$ bound states. The idea is to see whether it is possible, in this way, to recover the doubly bound analog (29) of the C3 continuum function. For $S$ bound states, we use the Hylleraas equation (see, e.g., [4]), i.e., the Schrödinger equation where Euler angles have been removed,

$$
\begin{gathered}
\left\{\left[-\frac{1}{2}\left(\frac{\partial^{2}}{\partial r_{1}^{2}}+\frac{2}{r_{1}} \frac{\partial}{\partial r_{1}}\right)+V_{1}\right]+\left[-\frac{1}{2}\left(\frac{\partial^{2}}{\partial r_{2}^{2}}+\frac{2}{r_{2}} \frac{\partial}{\partial r_{2}}\right)+V_{2}\right]\right. \\
+\left[-\left(\frac{\partial^{2}}{\partial r_{12}^{2}}+\frac{2}{r_{12}} \frac{\partial}{\partial r_{12}}\right)+V_{12}\right] \\
\left.+\left(-\hat{\mathbf{r}}_{1} \cdot \hat{\mathbf{r}}_{12} \frac{\partial}{\partial r_{1}}+\hat{\mathbf{r}}_{2} \cdot \hat{\mathbf{r}}_{12} \frac{\partial}{\partial r_{2}}\right) \frac{\partial}{\partial r_{12}}\right\} \Psi^{B}=E^{B} \Psi^{B},
\end{gathered}
$$

where we label $E^{B}$ the energy. The last term on the left-hand side involves mixed partial derivatives and introduces the nonseparability character of the system.

Because of the presence of vectors, we cannot start by writing the equivalent of relation (4). Without loss of generality, we start instead from the bound equivalent of relation (14), i.e.,

$$
\Psi^{B}=e^{-Z r_{1} / n_{1}} e^{-Z r_{2} / n_{2}} \varphi^{B}
$$

Upon substitution in Eq. (30), one gets the equivalent of Eq. (15)

$$
\begin{aligned}
\{[- & \left.\frac{1}{2}\left(\frac{\partial^{2}}{\partial r_{1}^{2}}+\frac{2}{r_{1}} \frac{\partial}{\partial r_{1}}\right)+\frac{Z}{n_{1}} \frac{1}{r_{1}}+\frac{Z}{n_{1}} \frac{\partial}{\partial r_{1}}+V_{1}\right] \\
+ & {\left[-\frac{1}{2}\left(\frac{\partial^{2}}{\partial r_{2}^{2}}+\frac{2}{r_{2}} \frac{\partial}{\partial r_{2}}\right)+\frac{Z}{n_{2}} \frac{1}{r_{2}}+\frac{Z}{n_{2}} \frac{\partial}{\partial r_{2}}+V_{2}\right] } \\
+ & {\left[-\left(\frac{\partial^{2}}{\partial r_{12}^{2}}+\frac{2}{r_{12}} \frac{\partial}{\partial r_{12}}\right)+V_{12}\right]+\left(-\frac{Z^{2}}{2 n_{1}^{2}}-\frac{Z^{2}}{2 n_{2}^{2}}-E^{B}+\right) } \\
& \times\left(-\hat{\mathbf{r}}_{1} \cdot \hat{\mathbf{r}}_{12} \frac{\partial}{\partial r_{1}}+\hat{\mathbf{r}}_{2} \cdot \hat{\mathbf{r}}_{12} \frac{\partial}{\partial r_{2}}\right) \frac{\partial}{\partial r_{12}} \\
& \left.+\left(\hat{\mathbf{r}}_{1} \cdot \hat{\mathbf{r}}_{12} \frac{Z}{n_{1}}-\hat{\mathbf{r}}_{2} \cdot \hat{\mathbf{r}}_{12} \frac{Z}{n_{2}}\right) \frac{\partial}{\partial r_{12}}\right\} \varphi^{B}=0 .
\end{aligned}
$$

We notice that the pure $r_{1}$ and $r_{2}$ parts are solved exactly by confluent hypergeometric functions ${ }_{1} F_{1}\left(1-n_{j}, 2, \frac{2 Z r_{j}}{n_{j}}\right) \quad(j$ $=1,2$ ), and therefore set

$$
\varphi^{B}={ }_{1} F_{1}\left(1-n_{1}, 2, \frac{2 Z r_{1}}{n_{1}}\right){ }_{1} F_{1}\left(1-n_{2}, 2, \frac{2 Z r_{2}}{n_{2}}\right) \chi^{B}
$$

so that

$$
\Psi^{B}=\varphi_{n_{1}}\left(r_{1}\right) \varphi_{n_{2}}\left(r_{2}\right) \chi^{B}
$$

Taking $E^{B}=-\frac{Z^{2}}{2 n_{1}^{2}}-\frac{Z^{2}}{2 n_{2}^{2}}$, we are left with

$$
\begin{aligned}
\{[- & \left.\left(\frac{\partial^{2}}{\partial r_{12}^{2}}+\frac{2}{r_{12}} \frac{\partial}{\partial r_{12}}\right)+V_{12}\right] \\
& +\left(-\hat{\mathbf{r}}_{1} \cdot \hat{\mathbf{r}}_{12} \frac{\partial}{\partial r_{1}}+\hat{\mathbf{r}}_{2} \cdot \hat{\mathbf{r}}_{12} \frac{\partial}{\partial r_{2}}\right) \frac{\partial}{\partial r_{12}} \\
& \left.+\left(\hat{\mathbf{r}}_{1} \cdot \hat{\mathbf{r}}_{12} \frac{Z}{n_{1}}-\hat{\mathbf{r}}_{2} \cdot \hat{\mathbf{r}}_{12} \frac{Z}{n_{2}}\right) \frac{\partial}{\partial r_{12}}\right\} \varphi^{B}=0 .
\end{aligned}
$$

This equation is the bound equivalent of Eq. (18) with the last two brackets identified as crossed and mixed terms, respectively. As in the continuum case, the $r_{12}$ dependence does not appear clearly. This difficulty is due to the choice of the starting function (31), exactly as when we used expression (14) for the continuum case. One has to be careful to check what terms are to be neglected. We recall that the continuum C3 is recovered from Eq. (18) when the action of operator $Q$ in Eq. (19) is neglected. Let us therefore consider only the $l=0$ contribution of operator $Q$, given its form (20b). We first replace the gradients by their radial components, i.e., $\boldsymbol{\nabla}_{12}=\hat{\mathbf{r}}_{12} \frac{\partial}{\partial r_{12}}$ and $\boldsymbol{\nabla}_{j}=\hat{\mathbf{r}}_{j} \frac{\partial}{\partial r_{j}}(j=1,2)$. Second, we take only the $l=0$ partial wave of ${ }_{1} F_{1}\left(1-i \alpha_{j}, 1, i\left(k_{j} r_{j}\right.\right.$ $\left.+\mathbf{k}_{j} \cdot \mathbf{r}_{j}\right)$ ), i.e., ${ }_{1} F_{1}\left(1-i \alpha_{j}, 2,2 i k_{j} r_{j}\right)$. Finally, we perform the analytic continuation $k_{j} \rightarrow i Z z_{j} / n_{j}$. We thus get the $S$ bound equivalent of operator $Q$,

$$
\begin{aligned}
Q^{B}= & \left\{{ }_{1} F_{1}\left(1-n_{1}, 2, \frac{2 Z r_{1}}{n_{1}}\right)\left[\left(-\frac{Z}{n_{2}} \hat{\mathbf{r}}_{2}-\frac{Z}{n_{2}} \hat{\mathbf{k}}_{2}\right)+\hat{\mathbf{r}}_{2} \frac{\partial}{\partial r_{2}}\right]\right. \\
& \times{ }_{1} F_{1}\left(1-n_{2}, 2, \frac{2 Z r_{2}}{n_{2}}\right)-{ }_{1} F_{1}\left(1-n_{2}, 2, \frac{2 Z r_{2}}{n_{2}}\right) \\
& \times\left[\left(-\frac{Z}{n_{1}} \hat{\mathbf{r}}_{1}-\frac{Z}{n_{1}} \hat{\mathbf{k}}_{1}\right)+\hat{\mathbf{r}}_{1} \frac{\partial}{\partial r_{1}}\right] \\
& \left.\times{ }_{1} F_{1}\left(1-n_{1}, 2, \frac{2 Z r_{1}}{n_{1}}\right)\right\} \cdot \hat{\mathbf{r}}_{12} \frac{\partial}{\partial r_{12}}
\end{aligned}
$$

Equation (34) can then be written as follows:

$$
\begin{aligned}
\left\{\frac{\varphi^{B}}{\chi^{B}}\right. & {\left[-\left(\frac{\partial^{2}}{\partial r_{12}^{2}}+\frac{2}{r_{12}} \frac{\partial}{\partial r_{12}}\right)-\left(\frac{Z}{n_{1}} \hat{\mathbf{k}}_{1}-\frac{Z}{n_{2}} \hat{\mathbf{k}}_{2}\right) \cdot \hat{\mathbf{r}}_{12} \frac{\partial}{\partial r_{12}}+V_{12}\right] } \\
& \left.+Q^{B}\right\} \chi^{B}=0
\end{aligned}
$$

which is the bound equivalent of Eq. (19). Hence by neglecting the action of $Q^{B}$ and setting $\kappa^{\prime}=\frac{1}{2}\left(\frac{Z}{n_{1}} \hat{\mathbf{k}}_{1}-\frac{Z}{n_{2}} \hat{\mathbf{k}}_{2}\right) \cdot \hat{\mathbf{r}}_{12}$, we obtain the following differential equation for $\chi^{B}$ : 


$$
\left[-\left(\frac{\partial^{2}}{\partial r_{12}^{2}}+\frac{2}{r_{12}} \frac{\partial}{\partial r_{12}}\right)-2 \kappa^{\prime} \frac{\partial}{\partial r_{12}}+V_{12}\right] \chi^{B}=0
$$

This is the $l=0$ equivalent of the differential equation for ${ }_{1} F_{1}\left(i \alpha_{12}, 1,-i\left(k_{12} r_{12}+\mathbf{k}_{12} \cdot \mathbf{r}_{12}\right)\right)$; the solution is simply $\chi^{B}$ $={ }_{1} F_{1}\left(\frac{1}{2 \kappa^{\prime}}, 2,2 \kappa^{\prime} r_{12}\right)$. Together with Eq. (33), we thus find the bound analog (29), i.e., formally, the same answer found when performing the analytic continuation of the C3 continuum function. The question remains, however, of how to relate $\kappa^{\prime}$ with the value $\kappa$ of Eq. (29) which itself is linked to $k_{12}$ defined by Eq. (7a).

To summarize, a C3 approach for $S$ bound states can be used to obtain the doubly bound analog of the $\mathrm{C} 3$ function, as long as one makes the same approximations as those made to obtain the double-continuum $\mathrm{C} 3$.

Because of its apparent similarity, it is also interesting to compare the present study with that which leads to Pluvinage wave function. The latter is the solution of Schrödinger equation (30) where the action of the mixed partial derivatives is ignored: in this case the differential equation becomes separable. The exact, diagonal, solution is the product of three $l$ $=0$ Coulomb solutions, one for each two-body problem (two attractive and one repulsive) $[4,5,25]$. Labeling by $n_{1}$ and $n_{2}$ the two bound functions, and taking a continuum Coulomb function corresponding to an energy $\kappa^{2}$, one obtains a Pluvinage-type wave function

$$
\begin{aligned}
\Psi_{P L U}^{B}\left(n_{1}, n_{2}, \kappa\right)= & N_{P L U}^{B}\left(n_{1}, n_{2}, \kappa\right) \varphi_{n_{1}}\left(r_{1}\right) \varphi_{n_{2}}\left(r_{2}\right) \\
& \times e^{-i \kappa r_{12}} F_{1}\left(1-\frac{i}{2 \kappa}, 2,2 i \kappa r_{12}\right),
\end{aligned}
$$

where $N_{P L U}^{B}\left(n_{1}, n_{2}, \kappa\right)$ is the normalization factor. By construction, this wavefunction satisfies Kato's cusp conditions. However, it corresponds to an energy $E_{P L U}^{B}=-\frac{Z^{2}}{2 n_{1}^{2}}-\frac{Z^{2}}{2 n_{2}^{2}}+\kappa^{2}$ which is not the energy $E^{B}$ of the C3 approach. Suppose now that, in searching the doubly bound analog, instead of the C3 we had started from the product of three continuum Coulomb wave functions $C(\alpha, \mathbf{k}, \mathbf{r})$, one for each two-body interaction, i.e., Eq. (8) with $D\left(\alpha_{12}, \mathbf{k}_{12}, \mathbf{r}_{12}\right)$ replaced by $C\left(\alpha_{12}, \mathbf{k}_{12}, \mathbf{r}_{12}\right)$. By performing the analytic continuation as done in Sec. III A, and proceeding in the same way we wrote Eq. (29), we would get exactly the function (38). In this sense, Pluvinage wave function is thus the doubly bound analog of the product of three Coulomb continuum wave functions, and not-as commonly believed (see, e.g., $[5,6,9]$ ) —of the C3 function.

As for the continuum case, let us make two more observations. If we neglect both the crossed and mixed terms in Eq. (34), we get the bound equivalent of Eq. (21), that is,

$$
\left[-\left(\frac{\partial^{2}}{\partial r_{12}^{2}}+\frac{2}{r_{12}} \frac{\partial}{\partial r_{12}}\right)+V_{12}\right] \chi^{B}=0
$$

The solution is then a $l=0$ Coulomb partial wave $\left.e^{-i k r_{12}{ }_{1} F_{1}\left(1-\frac{i}{2 k}, 2\right.}, 2 i k r_{12}\right)$ in the limit $k \rightarrow 0$, i.e., a Bessel function $I_{1}\left(2 \sqrt{r_{12}}\right) / \sqrt{r_{12}}$ [see Eq. (13.3.1) of [34]] which is exactly the $l=0$ partial wave term of the Bessel function (22), solution of Eq. (21). Collecting the results, we have

$$
\Psi^{B}=N^{B} \varphi_{n_{1}}\left(r_{1}\right) \varphi_{n_{2}}\left(r_{2}\right) \frac{I_{1}\left(2 \sqrt{r_{12}}\right)}{\sqrt{r_{12}}},
$$

where $N^{B}$ is the normalization factor. This wave function is nothing else than the $l=0$ partial wave term of the continuum wave function given by Eq. (23). It will appear again below, as a special case of $\Psi_{C 3}^{B}\left(n_{1}, n_{2}, \kappa\right)$, the doubly bound analog of the $\mathrm{C} 3$ continuum.

If on the other hand, we neglect the electron-electron interaction from the outset, one finds the independent particles model (see, e.g., [35]), i.e., Eq. (33) with no correlation function $\left(\chi^{B}=1\right)$ but with the same energy $E^{B}=-\frac{Z^{2}}{2 n_{1}^{2}}-\frac{Z^{2}}{2 n_{2}^{2}}$.

\section{BOUND ANALOG AND EQUIVALENT OF THE $\boldsymbol{r}_{12}$ DISTORTION FACTOR}

Let us look more closely at the analytical continuation of the distortion factor ${ }_{1} F_{1}\left(i \alpha_{12}, 2,-2 i k_{12} r_{12}\right)$ of relation (27). We shall proceed in two different ways. The first one (Sec. IV A) assumes that $k_{12}$ becomes imaginary $(i \kappa)$ by analytical continuation and hence the double-bound analog wave function (29) is used. While this approach is not mathematically strict, it has the merit of (i) yielding a rather simple function; (ii) allowing for a comparison with the Pluvinage wave function [8] in the case of the ground state. The second one (Sec. IV B) deals properly with the angular dependence of $k_{12}$, is mathematically rigorous, and gives the proper double-bound equivalent of $\mathrm{C} 3$.

\section{A. Simple approach: $k_{12}$ taken as a parameter}

By simple analytic continuation (rotation in the complex plane), one can replace $k_{12}$ by $i \kappa$, with $\kappa$ treated as a parameter. Indeed, as $k_{1}$ and $k_{2}$ become imaginary by analytical continuation $\left[k_{j} \rightarrow i Z z_{j} / n_{j}(j=1,2)\right]$, relations (7a) and (7c)though not used-suggest this transformation. In this case $\kappa$ is not linked in any way to $k_{1}, k_{2}$, and hence to $n_{1}, n_{2}$. The resulting double-bound analog wave function is given by Eq. (29). Since the correlation function $\chi^{B}$ of Eq. (37) gives no contribution to the energy, in agreement with the concept of distortion factor, the energy $E^{B}=-\frac{Z^{2}}{2 n_{1}^{2}}-\frac{Z^{2}}{2 n_{2}^{2}}$ is the same as that obtained for the independent particle model.

If we now focus on the ground state, $n_{1}=n_{2}=1$, within the model we get

$$
\Psi_{C 3}^{B}(1,1, \kappa)=N_{C 3}^{B}(1,1, \kappa) e^{-Z r_{1}-Z r_{2}}{ }_{1} F_{1}\left(\frac{1}{2 \kappa}, 2,2 \kappa r_{12}\right) .
$$

The confluent hypergeometric plays the role of correlation function and is always positive; the double-bound analog wave function $\Psi_{C 3}^{B}(1,1, \kappa)$ has therefore no nodes as it should be for the ground state of two-electron atoms. To relate $\Psi_{C 3}^{B}(1,1, \kappa)$ to the real three-body problem, a procedure to fix the arbitrary value $\kappa$ should be implemented. This can be done, for example, by optimizing the mean energy $\langle E\rangle$ of the full three-body Hamiltonian, Eq. (30), with respect to the variational parameter $\kappa$. In the case of helium $(Z=2)$, one 
finds $\langle E\rangle=-2.87854$ a.u. $\left[N_{C 3}^{B}(1,1,-0.255)=1.550\right]$ for $\kappa=$ -0.255 . This calculation (and all the others presented in this section) was performed with FORTRAN and MATHEMATICA codes, with an accuracy of up to five significative figures.

If we let $\kappa \rightarrow 0$, the first derivative in Eq. (37) is removed, and the hypergeometric function reduces to a Bessel function $I_{1}\left(2 \sqrt{r_{12}}\right) / \sqrt{r_{12}}$ [34]. The corresponding ground-state wave function reads

$$
\Psi_{C 3}^{B}(1,1, \kappa \rightarrow 0)=N_{C 3}^{B}(1,1, \kappa \rightarrow 0) e^{-Z r_{1}-Z r_{2}} \frac{I_{1}\left(2 \sqrt{r_{12}}\right)}{\sqrt{r_{12}}} .
$$

where, for helium, $N_{C 3}^{B}(1,1, \kappa \rightarrow 0)=1.45923$ and yields $\langle E\rangle=-2.87462$ a.u. Note that this wave function is the same as that given by Eq. (39) for $n_{1}=n_{2}=1$, obtained by neglecting the crossed and mixed terms in the $\mathrm{C} 3$ approach for $S$ bound states.

Because of its apparent similarity, let us compare $\Psi_{C 3}^{B}(1,1, \kappa)$ with Pluvinage's wave function (energy $E_{P L U}^{B}=$ $\left.-Z^{2}+\kappa^{2}\right)[8]$

$$
\begin{aligned}
\Psi_{P L U}^{B}(1,1, \kappa)= & N_{P L U}^{B}(1,1, \kappa) e^{-Z r_{1}-Z r_{2}} e^{-i \kappa r_{12}} \\
& { }_{1} F_{1}\left(1-\frac{i}{2 \kappa}, 2,2 i \kappa r_{12}\right),
\end{aligned}
$$

where the optimized parameter for helium $\kappa=0.41$ yields a ground-state mean energy of $\langle E\rangle=-2.8788$ a.u. $\left[N_{P L U}^{B}(1,1,0.41)=1.535\right]$. Note that, contrary to $\Psi_{C 3}^{B}(1,1, \kappa)$, the real function $\Psi_{P L U}^{B}(1,1, \kappa)$ presents an infinite number of unphysical nodes.

\section{B. Rigorous approach}

A more rigorous approach for the distortion factor ${ }_{1} F_{1}\left(i \alpha_{12}, 2,-2 i k_{12} r_{12}\right)$ consists in the following procedure. The presence of the scalar product $\mathbf{k}_{1} \cdot \mathbf{k}_{2}$ in Eq. (28) suggests using an expansion in Legendre polynomials,

$$
{ }_{1} F_{1}\left(i \alpha_{12}, 2,-2 i k_{12} r_{12}\right)=\sum_{l} g_{l}\left(k_{1}, k_{2}, r_{12}\right) \frac{2 l+1}{4 \pi} P_{l}\left(\hat{\mathbf{k}}_{1} \cdot \hat{\mathbf{k}}_{2}\right),
$$

where the coefficients are given by

$$
\begin{aligned}
g_{l}\left(k_{1}, k_{2}, r_{12}\right)= & \frac{2 \pi}{k_{1} k_{2}} \int_{\left|k_{1}-k_{2}\right|}^{k_{1}+k_{2}} F_{1}\left(\frac{i}{k}, 2,-i k r_{12}\right) \\
& \times P_{l}\left(\frac{k_{1}^{2}+k_{2}^{2}-k^{2}}{2 k_{1} k_{2}}\right) k d k .
\end{aligned}
$$

For $S$ states, we retain only the $l=0$ contribution in Eq. (43), that is,

$$
g_{0}\left(k_{1}, k_{2}, r_{12}\right)=\frac{2 \pi}{k_{1} k_{2}} \int_{\left|k_{1}-k_{2}\right|}^{k_{1}+k_{2}} F_{1}\left(\frac{i}{k}, 2,-i k r_{12}\right) k d k .
$$

We have thus removed the problem of the angle $\hat{\mathbf{k}}_{1} \cdot \hat{\mathbf{k}}_{2}$ in $k_{12}$; all possible $k_{12}$ values are taken into account through Eq.
(44). This is quite different from the previous approach where $k_{12}$ takes only one value. Since the function $g_{0}\left(k_{1}, k_{2}, r_{12}\right)$ depends now only on the modulus of $k_{1}$ and $k_{2}$, we can perform the analytic continuation, i.e., $k_{j}$ replaced by $-i Z / n_{j}(j=1,2)$. We thus obtain the integral

$$
g_{n_{1}, n_{2}}\left(r_{12}\right)=\frac{2 \pi n_{1} n_{2}}{Z^{2}} \int_{\left|Z / n_{1}-Z / n_{2}\right|}^{Z / n_{1}+Z / n_{2}} F_{1}\left(-\frac{1}{k}, 2,-k r_{12}\right) k d k,
$$

which can be easily evaluated numerically. Collecting the results, we get the doubly bound equivalent of the $\mathrm{C} 3$ continuum function,

$$
\Psi_{C 3}^{B}\left(n_{1}, n_{2}\right)=N_{C 3}^{B}\left(n_{1}, n_{2}\right) \varphi_{n_{1}}\left(r_{1}\right) \varphi_{n_{2}}\left(r_{2}\right) g_{n_{1}, n_{2}}\left(r_{12}\right) .
$$

Note that, like the doubly bound analog $\Psi_{C 3}^{B}\left(n_{1}, n_{2}, \kappa\right)$ [Eq. (29)], the double-bound equivalent wave function $\Psi_{C 3}^{B}\left(n_{1}, n_{2}\right)$ (i) satisfies Kato cusp conditions at all two-body coalescence points and (ii) has a correlation function which is a positive function. However, contrary to $\Psi_{C 3}^{B}\left(n_{1}, n_{2}, \kappa\right)$, the doubly bound equivalent $\Psi_{C 3}^{B}\left(n_{1}, n_{2}\right)$ is free of variational parameters.

Specifying $\Psi_{C 3}^{B}\left(n_{1}, n_{2}\right)$ for the ground state, we have

$$
\Psi_{C 3}^{B}(1,1)=N_{C 3}^{B}(1,1) e^{-Z r_{1}} e^{-Z r_{2}} \int_{0}^{2 Z}{ }_{1} F_{1}\left(-\frac{1}{k}, 2,-k r_{12}\right) k d k .
$$

For helium, the corresponding mean energy and normalization factor are, respectively, $\langle E\rangle=-2.86276$ a.u. and $N_{C 3}^{B}(1,1)=0.22196$.

The energy eigenvalue $E^{B}=-\frac{Z^{2}}{2 n_{1}^{2}}-\frac{Z^{2}}{2 n_{2}^{2}}$ associated with $\Psi_{C 3}^{B}\left(n_{1}, n_{2}\right)$ is the same as that of $\Psi_{C 3}^{B}\left(n_{1}, n_{2}, \kappa\right)$, Eq. (29), i.e., the energy corresponding to the independent particle model. Indeed, in both cases, the distortion factors do not contribute to the energy. However, they produce substantial differences when they are used to represent the real threebody Coulomb problem. This is already illustrated through the mean energies given above $\langle E\rangle$ when compared with that of the independent particle model $\langle E\rangle=-2.75$ a.u. The effect of correlation on the real three-body Coulomb problem will further appear below through the study of the local energy and through the dynamical tests discussed in Sec. V.

Let us now compare graphically the correlation functions found above, in the case of the ground state $\left(n_{1}=n_{2}=1\right)$ of helium. In Fig. 1, we have plotted $\Psi_{C 3}^{B} e^{Z\left(r_{1}+r_{2}\right)} / N_{C 3}^{B}$ (the normalization factor is omitted for the purpose of the comparison), for several $\Psi_{C 3}^{B}$, as a function of the electron-electron distance $r_{12}$. All of them have the linear behavior $1+r_{12} / 2$ near $r_{12}=0$ (Kato's cusp condition at the electron-electron coalescence), but differ as $r_{12}$ increases. We compare the results corresponding to $\Psi_{C 3}^{B}(1,1, \kappa=-0.255)$ [Eq. (40)], $\Psi_{P L U}^{B}(1,1, \kappa=0.41)$ [Eq. (42)], $\Psi_{C 3}^{B}(1,1, \kappa \rightarrow 0)$ [Eq. (41)] and $\Psi_{C 3}^{B}(1,1)$ [Eq. (46)]. As can be seen from the figure, all correlation functions-except for Pluvinage's-present similar behaviors (monotonic increase, with no nodes). For the first atomic units, however, also the Pluvinage correlation function of $\Psi_{P L U}^{B}(1,1, \kappa=0.41)$ presents a monotonic behavior, while its asymptotic behavior is quite different. 


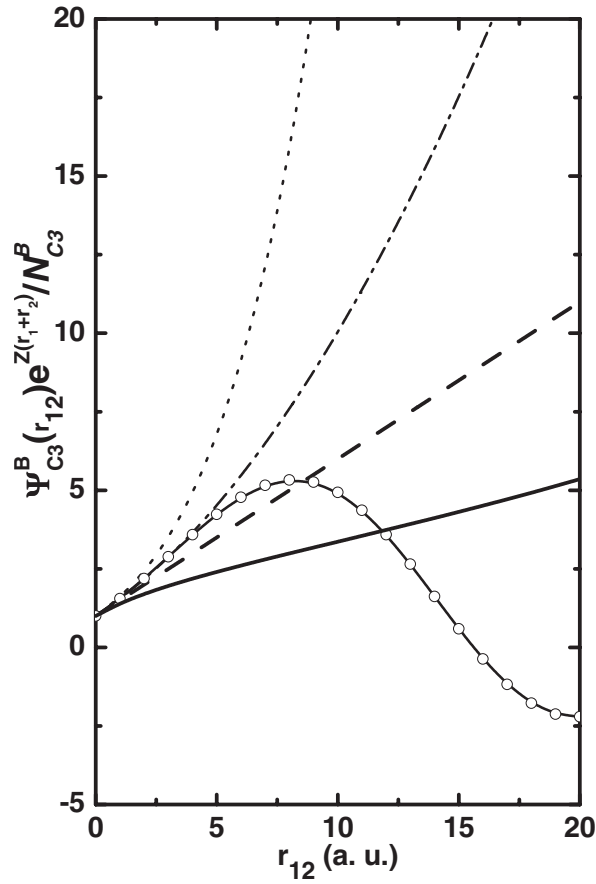

FIG. 1. Several correlation functions $\Psi_{C 3}^{B} e^{Z\left(r_{1}+r_{2}\right)} / N_{C 3}^{B}$ for the ground state of helium $(Z=2)$ are plotted vs the electron-electron distance $r_{12}$. The double-bound equivalent $\Psi_{C 3}^{B}(1,1)$, Eq. (46), is shown with a solid line, while Pluvinage $\Psi_{P L U}^{B}(1,1, \kappa=0.41)$ is shown with solid line plus open circles. Two double-bound analogs, corresponding to two choices of the parameter $\kappa$, are also presented: $\Psi_{C 3}^{B}(1,1, \kappa=-0.255)$ with a dot-dashed line and $\Psi_{C 3}^{B}(1,1, \kappa \rightarrow 0)$ with a dotted line. The function $1+r_{12} / 2$ suggested by Patil [36] is shown with a dashed line.

We may further compare the effect of the correlation functions through the analysis of the local energy (as done recently, for example, in Refs. $[4,5,38]$ ) which provides a very strong test of any trial wave function. Indeed, only for the exact wave function, the local energy defined as [37]

$$
E\left(r_{1}, r_{2}, r_{12}\right)=\frac{1}{\Psi\left(r_{1}, r_{2}, r_{12}\right)} H \Psi\left(r_{1}, r_{2}, r_{12}\right)
$$

will be a constant throughout the entire configuration space, i.e., an eigenvalue of Eq. (30), and hence equal to the mean energy [this is the case, for example, for the hydrogenic ions of nuclear charge $Z$, for which the local and mean energies are equal to $E=-Z^{2} / 2 n^{2}$ (a.u.)]. For any other trial wave function, the local energy will not be a constant value; the averaging over the whole space smooths out the variations of the local energy and provides a finite constant value. The variation with respect to the exact numerical value $\left(E_{\text {exact }}=-2.903724\right.$ a.u. in the case of helium [26]) may be represented by the quantity $\delta E=E\left(r_{1}, r_{2}, r_{12}\right)-E_{\text {exact }}$ : small values of $\delta E$ are then a sign that a wave function behaves well locally. For illustration purposes, we have chosen to take the six cuts of the three-dimensional space $\left(r_{1}, r_{2}, r_{12}\right)$ considered in Figs. 1 and 2 of Ref. [38]. In this way, the comparison can be easily extended to the functions considered in that paper. In Figs. 2 and 3 we have plotted the quantity $\delta E$ as a function of the mutual angle $\theta_{12}=\operatorname{arcos}\left[\left(r_{1}^{2}+r_{2}^{2}-r_{12}^{2}\right) /\left(2 r_{1} r_{2}\right)\right]$ for a selection of $\left(r_{1}, r_{2}\right)$ values. To avoid overcrowding the figures, we have included the results corresponding to the same wave functions as in Fig. 1, except for that of Patil. To facilitate the comparison, the same line symbols as in Fig. 1 are used, and the same vertical scale is used for the six panels. The observed variations of $\delta E$ with $\theta_{12}$ differ only through the chosen correlation functions. The three wave functions $\Psi_{C 3}^{B}(1,1, \kappa=$ $-0.255)$ [Eq. (40)], $\Psi_{P L U}^{B}(1,1, \kappa=0.41)$ [Eq. (42)], and $\Psi_{C 3}^{B}(1,1, \kappa \rightarrow 0)$ [Eq. (41)] are seen to be bunched together in all situations. On the other hand, the double-bound equivalent wave function $\Psi_{C 3}^{B}(1,1)$ [Eq. (46)] is separated from the previous ones. Since, by construction, all wave functions considered satisfy the electron-electron cusp condition, the local energy does not present any divergency near $\theta_{12}=0$ when $r_{1}=r_{2}$.

\section{DYNAMICAL TESTS FOR THE DOUBLE-BOUND WAVE FUNCTIONS}

The simultaneous excitation and ionization, as well as double ionization, of atoms by photon impact offer a series of dynamical tests for the double-bound wave functions $\Psi^{B}\left(\mathbf{r}_{1}, \mathbf{r}_{2}\right)$. The ejection or excitation of two electrons strongly depends on the electron-electron correlation of both initial and final states [12]. In particular in the high-energy nonrelativistic regime, the photoionization cross sections are closely linked to the Coulomb singularities of the electronnucleus and electron-electron interactions [12]. The photoabsorption might occur through the shake-off (SO) and quasifree $(\mathrm{QF})$ mechanisms. The first process corresponds to the case where one of the electrons is ejected with high energy while the second remains bound to the nucleus (ionizationexcitation) or leaving the atom with low velocity. The QF mechanism, on the other hand, is associated with the situation where both electrons share the absorbed photon without any participation of the nucleus. In both situations the cross sections are related to the correlation matrix defined by Suric et al. [12]

$$
M_{\lambda}=\left|\int d^{3} \mathbf{r}_{2} C_{\lambda}^{*}\left(\mathbf{r}_{2}\right) \Psi^{B}\left(0, \mathbf{r}_{2}\right)\right|^{2},
$$

where $C_{\lambda}\left(\mathbf{r}_{2}\right)$ defines a hydrogenic bound state $(\lambda$ $=\{n, l, m\})$ for ionization-excitation, or a continuum wave function $\left(\lambda=\mathbf{k}_{2}\right)$ for double ionization [12,39]. The shake-off ratio of double-to-single ionization cross section is given at high photon energies by

$$
R_{S O}=1-\sum_{n} R_{n},
$$

where

$$
R_{n}=\frac{M_{n}}{\int d^{3} \mathbf{r}_{2}\left|\Psi^{B}\left(0, \mathbf{r}_{2}\right)\right|^{2}},
$$

and the sum runs over all possible bound states of the residual ion. On the other hand, the total cross section for the 


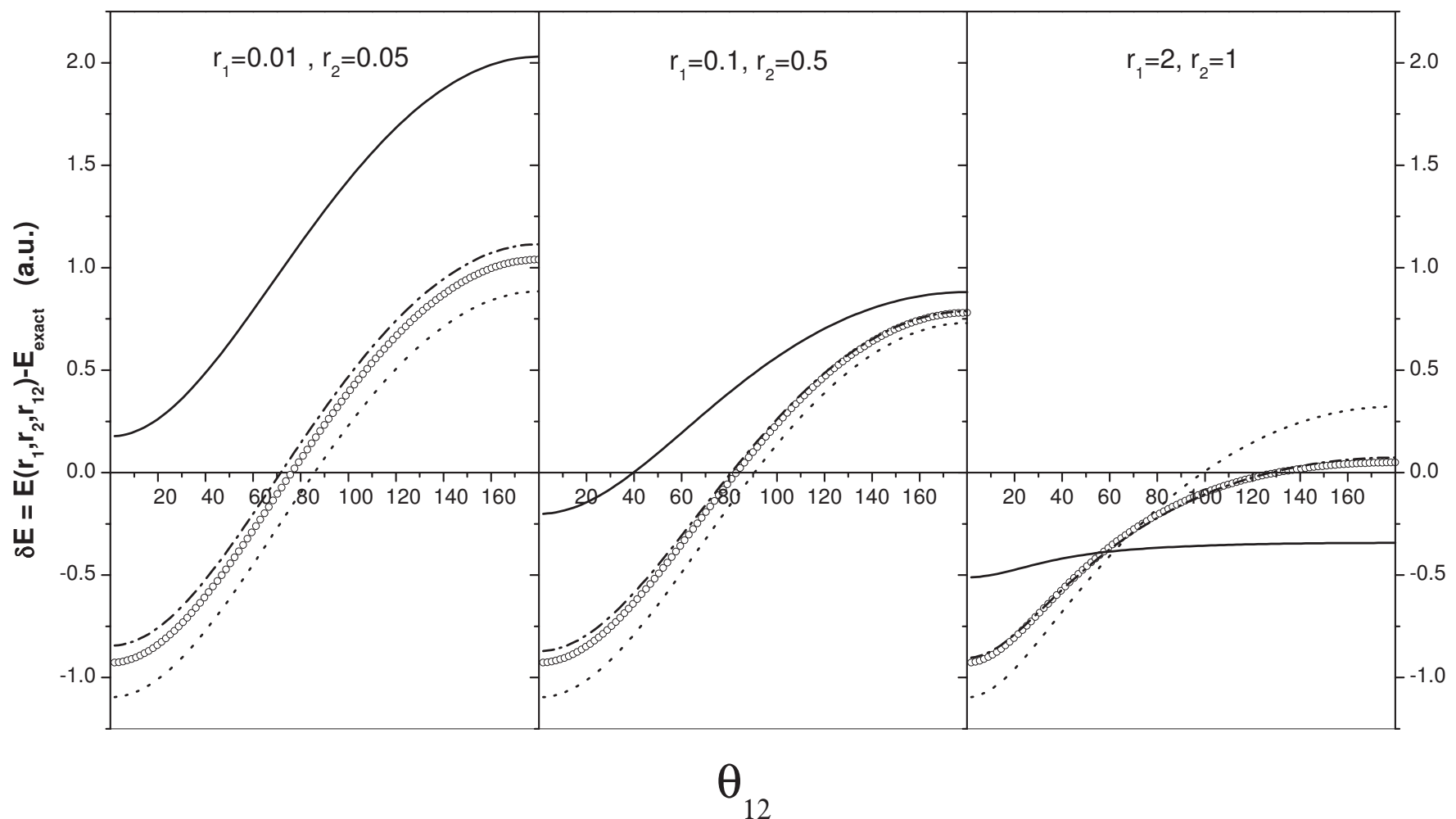

FIG. 2. The difference $\delta E=E\left(r_{1}, r_{2}, r_{12}\right)-E_{\text {exact }}$ calculated with four helium bound wave functions is plotted as a function of the mutual angle $\theta_{12}=\operatorname{arcos}\left[\left(r_{1}^{2}+r_{2}^{2}-r_{12}^{2}\right) /\left(2 r_{1} r_{2}\right)\right]$ for three pairs of $\left(r_{1}, r_{2}\right)$ values indicated in each panel (all with same vertical scale). The same line symbols as in Fig. 1 are used, i.e., $\Psi_{C 3}^{B}(1,1)$ with a solid line, $\Psi_{P L U}^{B}(1,1, \kappa=0.41)$ with a solid line plus open circles, $\Psi_{C 3}^{B}(1,1, \kappa=$ $-0.255)$ with a dot-dashed line, and $\Psi_{C 3}^{B}(1,1, \kappa \rightarrow 0)$ with a dotted line.

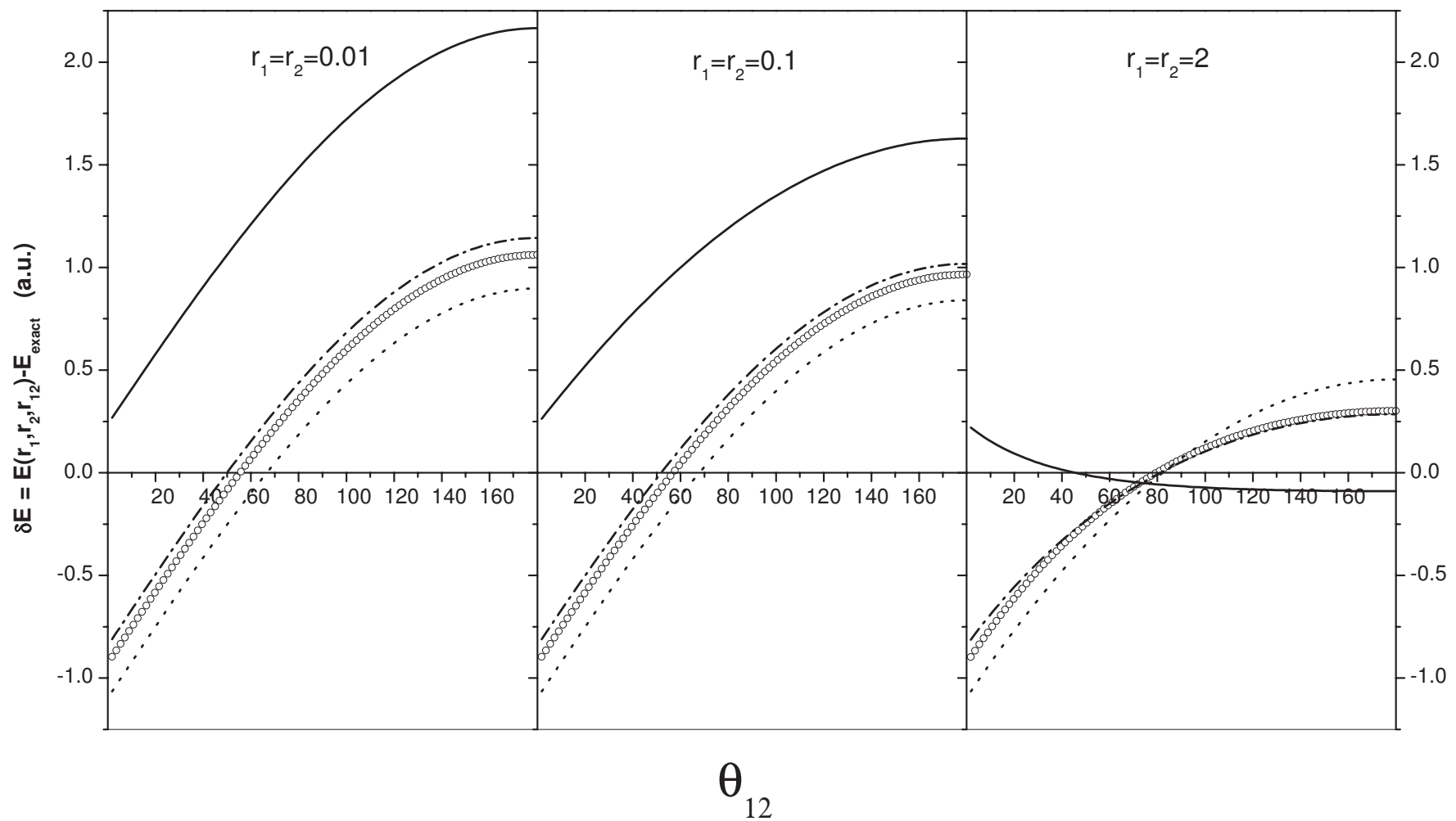

FIG. 3. Same as Fig. 2, but for three other pairs of $\left(r_{1}, r_{2}\right)$ values. 
TABLE I. Ratios $R_{n}$-in relative percentages - of the single to total ionization for photoabsorption by helium, for $n=1-5$. The present results correspond to the functions $\Psi_{C 3}^{B}(1,1, \kappa=-0.255), \Psi_{C 3}^{B}(1,1, \kappa \rightarrow 0)$, and $\Psi_{C 3}^{B}(1,1)$. For comparison, are included the values reported by Dalgarno and Sadeghpour [43] obtained with the 20-parameter Hylleraas wave function $\Psi_{H}^{(20)}$, and by Jones et al. [5] obtained with the Pluvinage wave function $\Psi_{P L U}^{B}(1,1, \kappa)$.

\begin{tabular}{cccccc}
\hline \hline & $\Psi_{H}^{(20)}$ & $\Psi_{P L U}^{B}(1,1, \kappa)$ & $\Psi_{C 3}^{B}(1,1, \kappa=-0.255)$ & $\Psi_{C 3}^{B}(1,1, \kappa \rightarrow 0)$ & $\Psi_{C 3}^{B}(1,1)$ \\
\hline 1 & 92.94 & 96.75 & 96.88 & 95.78 & 98.68 \\
2 & 4.45 & 1.46 & 1.416 & 2.15 & 0.47 \\
3 & 0.55 & 0.27 & 0.25 & 0.35 & 0.095 \\
4 & 0.18 & 0.10 & 0.092 & 0.12 & 0.036 \\
5 & 0.08 & 0.05 & 0.044 & 0.06 & 0.017 \\
\hline \hline
\end{tabular}

double ionization through the quasifree mechanism is found to be proportional to [12]

$$
\sigma_{Q F} \propto \int d^{3} \mathbf{r}\left|\Psi^{B}(\mathbf{r}, \mathbf{r})\right|^{2} .
$$

As explained by Drukarev [40], the correction to the $R_{S O}$ due to the quasifree mechanism is related to the quantity $C$ given by

$$
C=\frac{\int d^{3} \mathbf{r}\left|\Psi^{B}(\mathbf{r}, \mathbf{r})\right|^{2}}{M_{1}},
$$

where $M_{1}$ corresponds to the evaluation of $M_{\lambda}$ with $\lambda$ $=\{1,0,0\}$.

The expression defining the matrix $M_{\lambda}$ is obtained by taking the high-energy limit of the complete transition matrix for single or double photoionization and using the electronnucleus Kato cusp conditions. Thus the photoabsorption process probes the double-bound wavefunction $\Psi^{B}\left(\mathbf{r}_{1}, \mathbf{r}_{2}\right)$ at the origin of the coordinate of the fast emitted electron. $M_{\lambda}$ is given by the sudden approximation, i.e., by projecting the initial-state wave function onto the final state of the shaken-up electron. The quasifree mechanism, on the other hand, is associated to the electron-electron cusp condition; it thus tests a different part of the configuration space, the region where the electrons are close to each other.

Compton scattering provides another test for the bound wave function $\Psi^{B}\left(\mathbf{r}_{1}, \mathbf{r}_{2}\right)$. Indeed, the ratio of double to total ionization cross section corresponding to high photon energy is given by [41]

$$
R_{C}=1-\sum_{n, l, m} \sigma_{n, l, m},
$$

where

$$
\sigma_{n, l, m}=\int d^{3} \mathbf{r}_{1}\left|\int d^{3} \mathbf{r}_{2} C_{n, l, m}^{*}\left(\mathbf{r}_{2}\right) \Psi^{B}\left(\mathbf{r}_{1}, \mathbf{r}_{2}\right)\right|^{2} .
$$

Contrary to $M_{\lambda}$ where the bound wave function appears only through it's form $\Psi^{B}\left(0, \mathbf{r}_{2}\right), \sigma_{n, l, m}$ is an average over all the coordinates $\mathbf{r}_{1}$, thus testing the wave function on the whole configuration space.

We have calculated several of these quantities in the case of helium. In Tables I-III, we give the results obtained with the bound wave functions: $\Psi_{C 3}^{B}(1,1, \kappa=-0.255)$ [Eq. (40)], $\Psi_{C 3}^{B}(1,1, \kappa \rightarrow 0)$ [Eq. (41)], and $\Psi_{C 3}^{B}(1,1)$ [Eq. (46)]. We shall compare them with those obtained with Pluvinage's wave function $\Psi_{P L U}^{B}(1,1, \kappa)$ [Eq. (42)] and with the 20 parameters, highly correlated, Hylleraas function of Hart and Herzberg [42], noted here $\Psi_{H}^{(20)}$. The results corresponding to Pluvinage's function are taken, except for $C$, from Ref. [5].

In Table I, we give the results-in relative percentagesobtained for the ratios $R_{n}$, leaving the residual ion in the $n s$ state $(n=1-5)$. One observes that of the three functions studied in this paper, it is $\Psi_{C 3}^{B}(1,1, \kappa \rightarrow 0)$ which gives the best results when compared to the benchmark values obtained with $\Psi_{H}^{(20)}$ and reported by Dalgarno and Sadeghpour [43]. In the independent particle model only $R_{1}$ contributes to photoabsorption; indeed, the contribution of all other $n s$ states vanishes by orthogonality (see definition of $M_{\lambda}$ ). Since the bound wave functions introduced in this paper have only angular correlation, the quantities $R_{n}$ we have presented are just testing the angular correlation factors.

In Table II, we present the results obtained for $C$. In this case $\Psi_{P L U}^{B}(1,1, \kappa), \quad \Psi_{C 3}^{B}(1,1, \kappa=-0.255)$ and $\Psi_{C 3}^{B}(1,1, \kappa$ $\rightarrow 0$ ) wave functions are giving similar results to that corresponding to $\Psi_{H}^{(20)}$ that we have calculated for comparison. We may again compare the results with that corresponding to the independent particle model. The value $C_{I P M}=1 / 8$ reported by Drukarev [40] is far from the values obtained with

TABLE II. Calculated values for the quantity $C$ defined by Eq. (52). The results corresponding to $\Psi_{C 3}^{B}(1,1, \kappa=-0.255), \Psi_{C 3}^{B}(1,1, \kappa \rightarrow 0)$, and $\Psi_{C 3}^{B}(1,1)$ are compared with our calculations for the Pluvinage and the 20-parameter Hylleraas wavefunctions.

\begin{tabular}{cccccc}
\hline \hline & $\Psi_{H}^{(20)}$ & $\Psi_{P L U}^{B}(1,1, \kappa)$ & $\Psi_{C 3}^{B}(1,1, \kappa=-0.255)$ & $\Psi_{C 3}^{B}(1,1, \kappa \rightarrow 0)$ & $\Psi_{C 3}^{B}(1,1)$ \\
\hline $\mathrm{C}$ & 0.0636 & 0.0626 & 0.0633 & 0.0599 & 0.0731 \\
\hline \hline
\end{tabular}


TABLE III. Ratios $\sigma_{n, 0,0}$-in relative percentages - of the single to total ionization for Compton scattering of a photon off helium, leaving the residual ion in the $n s$ state indicated. The present results correspond to the functions $\Psi_{C 3}^{B}(1,1, \kappa=-0.255), \Psi_{C 3}^{B}(1,1, \kappa \rightarrow 0)$, and $\Psi_{C 3}^{B}(1,1)$. For comparison, we have included the values reported by Suric et al. [41] obtained with $\Psi_{H}^{(20)}$, and those obtained with $\Psi_{P L U}^{B}(1,1, \kappa)$ reported by Jones et al. [5].

\begin{tabular}{lccccc}
\hline \hline$n l$ & $\Psi_{H}^{(20)}$ & $\Psi_{P L U}^{B}(1,1, \kappa)$ & $\Psi_{C 3}^{B}(1,1, \kappa=-0.255)$ & $\Psi_{C 3}^{B}(1,1, \kappa \rightarrow 0)$ & $\Psi_{C 3}^{B}(1,1)$ \\
\hline $1 s$ & 96.00 & 97.15 & 97.22 & 95.99 & 98.98 \\
$2 s$ & 2.49 & 0.78 & 0.77 & 1.23 & 0.23 \\
$3 s$ & 0.28 & 0.14 & 0.13 & 0.19 & 0.04 \\
$4 s$ & 0.09 & 0.05 & 0.05 & 0.065 & 0.02 \\
\hline \hline
\end{tabular}

our correlated functions: the difference can thus be attributed directly to the angular correlation factors.

Finally, in Table III, we give the results-in relative percentages - obtained for $\sigma_{n, l, m}$ for $n s$ states $(n=1,2,3,4)$. In this case, again, the function $\Psi_{C 3}^{B}(1,1, \kappa \rightarrow 0)$ is showing the best agreement when compared with the benchmark values obtained with $\Psi_{H}^{(20)}$ and reported in Ref. [41].

Of the three functions introduced in Sec. IV, $\Psi_{C 3}^{B}(1,1, \kappa$ $\rightarrow 0$ ) yields the overall best results for the quantities $R_{n}, C$, and $\sigma_{n, l, m}$ when compared to reference values. Surprisingly, the description given for $\sigma_{n, l, m}$, which tests the function over the whole configuration space, is also quite good. This is related to the fact that the function $\Psi_{C 3}^{B}(1,1, \kappa \rightarrow 0)$ gives a distribution of probability quite similar to that of the highly correlated $\Psi_{H}^{(20)}$, in spite of not including any radial correlation. One has to be careful, though, in drawing conclusions. The dynamical tests discussed above are, by no means, conclusive on the quality of a bound-state wave functions. Indeed, it is worth reminding that the double-bound analog and equivalent wave functions studied yield rather bad mean ground-state energies thus indicating that much of the correlation is missing.

\section{SUMMARY AND PERSPECTIVES}

In this paper we have studied the double-bound analog for $S$ states of the doubly continuum C3 wave function. We have first briefly discussed the way to obtain the doublecontinuum $\mathrm{C} 3$ in order to identify the terms neglected from the full three-body Hamiltonian. In this first part, we have given the Hamiltonian satisfied by the C3 function, and underlined the fact that the momentum $\mathbf{k}_{12}=\left(\mathbf{k}_{1}-\mathbf{k}_{2}\right) / 2$ should be considered altogether with the Schrödinger equation in order to get the correct function.

The doubly bound analog for $S$ states is given by the wave function $\Psi_{C 3}^{B}\left(n_{1}, n_{2}, \kappa\right)$ [Eq. (29)], and not-as commonly believed-by the Pluvinage wave function [Eq. (38)]. The result is obtained with two different procedures. The first one is based on the double analytic continuation of the continuum C3 wave function. This method allows us to ensure that we are dealing with the correct bound version of the C3 double continuum. Alternatively, starting from Hylleraas Hamiltonian and identifying the terms equivalent to those neglected in the double-continuum case, the double-bound analog is formally recovered. In both procedures, we are left with an undetermined quantity which represents the magnitude of the relative momentum $k_{12}$, or its analytic continuation $\kappa$. For bound states, one may consider it as a variational parameter to be determined by minimizing the mean energy corresponding to the full three-body Hamiltonian. A rigorous treatment (using a full expansion in terms of angular functions followed by an analytic continuation of the electronnucleus momenta) allowed us to find the double-bound equivalent of the C3 wave function, given by Eq. (45). The result does not depend on any quantization axis as physically expected and is a parameter-free wave function. Like for the C3 continuum, by construction, both the double-bound ana$\log (29)$ and equivalent [Eq. (45)] wave functions satisfy Kato cusp conditions at all two-body coalescence points as can be observed from the study of the local energy performed in Sec. IV B.

While the energy corresponding to the double-bound C3 wave function is the same as that corresponding to the independent particle approach, the distortion factors lead to a much better mean energy of the full three-body Hamiltonian. This issue will be further discussed in a separate paper, where different basis sets constructed with double-bound C3 functions will be investigated. Both ground and excited states of He-like systems will be studied.

Different dynamical tests to the wave functions introduced here have been performed. They are associated to cross sections corresponding to photoabsorption and to Compton scattering. The values for $R_{n}, C$, and $\sigma_{n, l, m}$ of Eqs. (50), (52), and (54), respectively, calculated with the wave function $\Psi_{C 3}^{B}(1,1, \kappa \rightarrow 0)$ (special case of the double-bound analog of C3) present the best general agreement when compared with the highly correlated 20-parameter Hylleraas function of Hart and Herzberg.

The study presented in this paper addressed the twoelectron problem case. The generalization to the case of $\mathrm{N}$-electron systems is straightforward. The analytic continuation used in Sec. III A can be applied to the $C N$ wave function, a generalization of the $\mathrm{C} 3$ to $N$ particles as described by Berakdar (see, e.g, [1]). By following the same steps, the $N$-bound analog for $S$ states of the $C N$ continuum wave function is then the product of $N$ Coulomb bound states multiplied by $M=N(N-1) / 2$ Coulomb distortion factors, 


$$
\begin{aligned}
\Psi_{C N}^{B}= & \prod_{m>l, j=1}^{N}\left[e^{-\left(Z / n_{j}\right) r_{j}} F_{1}\left(1-n_{j}, 2,2 \frac{Z}{n_{j}} r_{j}\right)\right] \\
& \times\left[{ }_{1} F_{1}\left(\frac{1}{2 \kappa_{l m}}, 2,2 \kappa_{l m} r_{l m}\right)\right],
\end{aligned}
$$

and involves $M$ parameters $\kappa_{l m}$. The extension to general masses is straightforward.

Since we have now in our hands a symmetric description of correlated bound and continuum wave functions, it would be very interesting to investigate their effect in the study of double ionization processes.

\section{ACKNOWLEDGMENTS}

One of the authors, G.G., would like to acknowledge the support by PICTR 2003/00437 of the ANPCYT (Argentina), PICT 5595 of the CONICET (Argentina), and PGI 24/F027 Universidad Nacional del Sur (Argentina).
[1] J. Berakdar, A. Lahmam-Bennani, and C. Dal Cappello, Phys. Rep. 374, 91 (2003).

[2] J. S. Briggs and V. Schmidt, J. Phys. B 33, R1 (2000).

[3] A. S. Kheifets and I. Bray, Phys. Rev. A 69, 050701(R) (2004).

[4] L. U. Ancarani, T. Montagnese, and C. Dal Cappello, Phys. Rev. A 70, 012711 (2004).

[5] S. Jones, J. H. Macek, and D. H. Madison, Phys. Rev. A 70, 012712 (2004).

[6] S. Jones and D. H. Madison, Phys. Rev. Lett. 91, 073201 (2003)

[7] C. R. Garibotti and J. E. Miraglia, Phys. Rev. A 21, 572 (1980); M. Brauner, J. Briggs, and H. Klar, J. Phys. B 22, 2265 (1989).

[8] P. Pluvinage, Ann. Phys. (Paris) 5, 145 (1950); J. Phys. Radium 12, 789 (1951).

[9] J. R. Götz, M. Walter, and J. S. Briggs, J. Phys. B 38, 1569 (2005).

[10] N. Stolterfoht, R. D. Dubois, and R. D. Rivarola, Electron Emission in Heavy Ion-Atom Collisions (Springer, Berlin, 1997).

[11] T. Kato, Commun. Pure Appl. Math. 10, 151 (1957).

[12] T. Suric, E. G. Drukarev, and R. H. Pratt, Phys. Rev. A 67, 022710 (2003).

[13] S. Jetzke and F. H. M. Faisal, J. Phys. B 25, 1543 (1992); S. Jetzke, J. Zeremba, and F. H. M. Faisal, Z. Phys. D: At., Mol. Clusters 11, 63 (1989).

[14] E. O. Alt and A. M. Mukhamedzhanov, Phys. Rev. A 47, 2004 (1993)

[15] J. Berakdar and J. S. Briggs, Phys. Rev. Lett. 72, 3799 (1994); J. Phys. B 27, 4271 (1994).

[16] J. Berakdar, Phys. Rev. A 53, 2314 (1996).

[17] F. D. Colavecchia, G. Gasaneo, and C. R. Garibotti, Phys. Rev. A 57, 1018 (1998).

[18] S. Otranto and C. R. Garibotti, Eur. Phys. J. D 27, 215 (2003).

[19] G. Gasaneo, S. Otranto, and K. V. Rodriguez, Proceedings of the XXIV International Conference on Photonic, Electronic and Atomic Collisions, Rosario, Argentina, 2005, edited by P. D. Fainstein, M. A. P. Lima, J. E. Miraglia, E. C. Montenegro, and R. D. Rivarola (World Scientific, Singapore, 2006).

[20] L. U. Ancarani, C. Dal Cappello, and T. Montagnese, in Ion- ization, Correlation, and Polarization in Atomic Collisions, edited by A. Lahmam-Bennani and B. Lohmann, AIP Conf. Proc. No. 811 (AIP, Melville, NY, 2006), p. 1.

[21] A. Lahmam-Bennani et al., Phys. Rev. A 59, 3548 (1999).

[22] S. Elazzouzi et al., J. Phys. B 38, 1391 (2005).

[23] L. U. Ancarani, T. Montagnese and C. Dal Cappello, in Electron and Photon Impact Ionization and Related Topics, edited by B. Piraux, IOP Conf. Proc. No. 183 (Institute of Physics, London, 2005), p. 21.

[24] F. D. Colavecchia, G. Gasaneo, and K. V. Rodriguez, J. Electron Spectrosc. Relat. Phenom.(to be published).

[25] L. U. Ancarani and C. Dal Cappello, J. Electron Spectrosc. Relat. Phenom. (to be published).

[26] V. I. Korobov, Phys. Rev. A 61, 064503 (2001).

[27] J. Berakdar, Phys. Rev. Lett. 78, 2712 (1997).

[28] G. Gasaneo, F. D. Colavecchia, C. R. Garibotti, J. E. Miraglia, and P. Macri, Phys. Rev. A 55, 2809 (1997).

[29] G. Gasaneo, F. D. Colavecchia, and C. R. Garibotti, J. Phys. B 30, L265 (1997).

[30] P. A. Macri, J. E. Miraglia, C. R. Garibotti, F. D. Colavecchia, and G. Gasaneo, Phys. Rev. A 55, 3518 (1997).

[31] D. P. Dewangan, J. Phys. B 30, L467 (1997).

[32] J. Berakdar, Phys. Rev. A 58, R1641 (1998).

[33] C. R. Garibotti, G. Gasaneo, and F. D. Colavecchia, Phys. Rev. A 62, 022710 (2000).

[34] M. Abramowitz and I. A. Stegun, Handbook of Mathematical Functions (Dover, New York, 1972).

[35] B. H. Bransden and C. J. Joachain, Physics of Atoms and Molecules, 2nd ed, (Prentice-Hall, Englewood Cliffs, NJ, 2003).

[36] S. H. Patil, Eur. J. Phys. 25, 91 (2004).

[37] J. H. Bartlett, J. J. Gibbons, and C. G. Dunn, Phys. Rev. 47, 679 (1935).

[38] K. V. Rodriguez and G. Gasaneo, J. Phys. B 38, L259 (2005).

[39] L. Andersson and J. Burgdörfer, Phys. Rev. A 50, R2810 (1994).

[40] E. G. Drukarev, Phys. Rev. A 51, R2684 (1995).

[41] T. Suric, K. Pisk, B. A. Logan, and R. H. Pratt, Phys. Rev. Lett. 73, 790 (1994).

[42] J. F. Hart and G. Herzberg, Phys. Rev. 106, 79 (1957).

[43] A. Dalgarno and H. R. Sadeghpour, Phys. Rev. A 46, R3591 (1992). 